\title{
A Rare Case of Bilateral Non-Hodgkin's Lymphoma of the Adrenal Gland
}

\author{
Maria Patricia G. Puno, ${ }^{1}$ Sheila Cagadas, ${ }^{2}$ Michael L. Villa ${ }^{1}$ \\ ${ }^{1}$ Section of Endocrinology and Metabolism, Department of Medicine, The Medical City, Pasig City, Philippines \\ ${ }^{2}$ Section of Clinical Pathology, The Medical City, Pasig City, Philippines
}

\begin{abstract}
Bilateral Adrenal Lymphoma is a rare disease which, when it is discovered, may be associated with adrenal insufficiency. We report a case of an 81-year-old female, with no known co-morbidities, presenting with anorexia and flank pain. Initial CT imaging revealed bilateral adrenal masses measuring $6.4 \times 7.4 \mathrm{~cm}$ on the right and $5.9 \times 5.8 \mathrm{~cm}$ on the left, with subsequent scans showing the masses to be rapidly enlarging. Aspiration biopsy revealed Non-Hodgkin's Lymphoma, diffuse large B cell type. She underwent chemotherapy with almost complete resolution of the adrenal tumors.
\end{abstract}

Keywords: primary adrenal lymphoma, adrenal biopsy, adrenal insufficiency, adrenal mass.

\section{INTRODUCTION}

The number of adrenal incidentalomas have been increasing in recent years due to the emergence of precise radiologic imaging techniques which may have been requested for the work-up of other organs in the body such as the abdomen or the thorax. Unexpected adrenal masses, when found, compel the clinician to evaluate them further for hormonal dysfunction and to determine if it is malignant.

The adrenal gland is a frequent site of involvement in disseminated lymphoma and other neoplasms, often occurring unilaterally in metastatic disease. Primary tumors from the adrenal gland occur with less frequency, making it difficult to establish treatment guidelines. In a descriptive study on the clinicopathologic profile of Filipino patients with malignant lymphomas, the adrenal gland was cited as a rare extranodal location for Non-

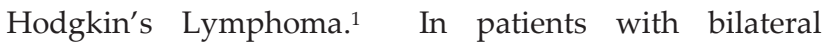
enlargement of the adrenal glands, a primary adrenal lymphoma should be considered. ${ }^{2}$ This condition occurs predominantly in males, often affects the elderly, and in about $70 \%$, affects both adrenals. ${ }^{3}$ Literature review reported only 116 cases of primary bilateral adrenal lymphoma worldwide, with only 26 cases of NonHodgkin's Lymphoma of Diffuse Large Cell B type reported in English literature. ${ }^{3-5}$ Some patients with primary bilateral adrenal lymphoma also show clinical or laboratory signs of adrenal insufficiency, which are not usually seen in unilateral adrenal NHL. ${ }^{6}$
The objectives of this case report are: 1) to present a unique case of a bilateral primary lymphoma of the adrenal glands, 2) to review the approach to the diagnosis and management of adrenal masses and primary adrenal lymphoma, and 3) to review related literature of and compare our case to published data and current guidelines.

\section{CASE}

An 81-year-old female with no medical comorbidities, initially sought consult for right flank pain accompanied with anorexia, easy fatigability and urinary frequency. A physician requested a urinalysis revealing microscopic hematuria. A subsequent CT scan incidentally revealed bilateral adrenal masses measuring $6.4 \times 2.7 \times 7.4 \mathrm{~cm}$ on the right and $5.9 \times 2.6 \times 5.8 \mathrm{~cm}$ on the left. A repeat CT scan a month later, showed the masses to be increasing in size with the mass on the right measuring $7.3 \times 4 \times 7.9 \mathrm{~cm}$, and that on the left measuring $8 \times 9.2 \times 5.2 \mathrm{~cm}$ (Figure 11). Functional work up included: Plasma renin activity of $1.540 \quad(0.5-1.9 \mathrm{ng} / \mathrm{ml} / \mathrm{hr})$, plasma aldosterone concentration of $3.846(1.0-16 \mathrm{ng} / \mathrm{dl}), 24$ hour urine cortisol of 90.36 (4.3 - $176 \mathrm{ug} / 24 \mathrm{hrs})$, and 24 hour urine catecholamines of $54.81(0-275 \mathrm{ug} / 24 \mathrm{hrs})$ which were all within normal range. Considering the masses could be metastases, a primary malignancy was likewise investigated. Chest x-ray was unremarkable, CA19-9 24.31 (NV $0-39 \mathrm{U} / \mathrm{ml}$ ) and alpha-fetoprotein 1.95 (NV <8.6 $\mathrm{ng} / \mathrm{ml}$ ) were both within normal ranges. A bone scan was done revealing findings in the vertebral column consistent with degenerative/osteoarthritic changes and 
indeterminate for osseous metastasis. Total calcium was $8.0 \mathrm{mg} / \mathrm{dl}$, with a corrected total serum calcium of 9.624 $\mathrm{mg} / \mathrm{dl}$ (NV: 8.8 - 10.2), phosphorus was $2.85 \mathrm{mg} / \mathrm{dl}$ (NV 2.69-4.49), and vitamin D was low at $21.8 \mathrm{ng} / \mathrm{ml}$ (NV 3040). Patient was noted to be persistently hyponatremic. An ACTH $(250 \mathrm{mcg})$ stimulation test revealed a baseline ACTH level of $111 \mathrm{pg} / \mathrm{ml}$ (NV 5-46), with serum cortisol levels of $6.1 \mu \mathrm{g} / \mathrm{dl}, 9.5 \mu \mathrm{g} / \mathrm{dl}$ and $6.6 \mathrm{ug} / \mathrm{dl}$ at baseline, 30 and 60 minutes after induction of $250 \mathrm{mcg}$ of ACTH respectively, indicating an inadequate adrenal reserve for which prednisone was started.
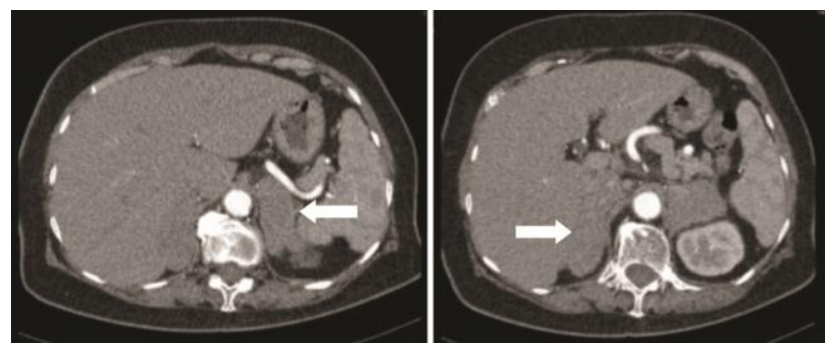

Figure 11. Contrast enhanced CT scan of the left (a) and right (b) adrenal gland before treatment

A CT scan guided aspiration biopsy of the masses was then done with a histopathologic finding of a malignant, small round cell tumor involving both adrenal glands, immunomorphologically compatible with a NonHodgkin's Lymphoma, Diffuse, Large B-cell type with a positive immunohistochemistry stain for LCA/CD45 and CD20. An elevated LDH IFCC 632.10 (NV: 135 - 225 U/L) and elevated $\beta 2$ microglobulin $4479 \mu \mathrm{g} / \mathrm{L}$ (NV: 830-1150) supported the diagnosis of Non-Hodgkin's Lymphoma.

Patient was started on a modified CHOP therapy (Cyclophosphamide, Vincristine, Prednisone, not including Doxorubicin) followed by radiation therapy to the adrenals. Rituximab treatment was likewise offered, however, the patient declined due to its cost.

A repeat CT scan of the adrenals done after 6 cycles of chemotherapy--which was done at 3 to 4 week intervals followed by 8 sessions of radiation therapy directed to the adrenals--showed a marked resolution of the bilateral adrenal masses, with the right adrenal gland exhibiting normal limb thickness and the left adrenal gland having a subcentimeter nodule measuring $0.6 \times 0.7 \mathrm{~cm}$ (Figure 12).
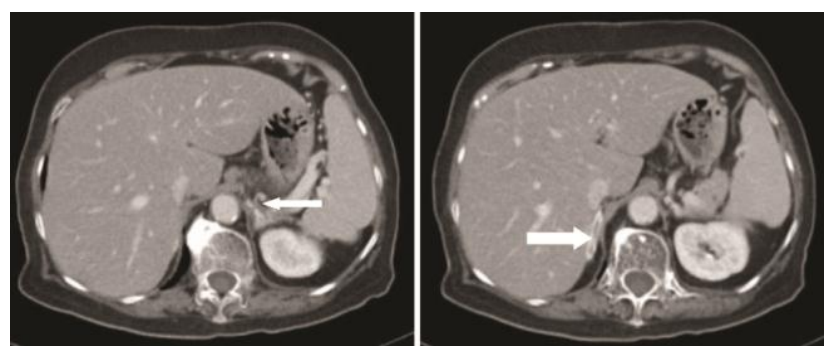

Figure 12. Venous Phase Contrast CT scan of the left (a) and right (b) adrenal gland after 6 cycles of chemotherapy
Eight months after initial diagnosis, and 2 months after chemotherapy and radiation therapy was completed, the patient sustained head trauma after a fall. A CT scan followed by an MRI of the brain showed multiple intracranial lesions consistent with brain metastases. She subsequently underwent palliative whole brain radiotherapy. The patient's general condition progressively deteriorated and she later succumbed to the disease 13 months after initial diagnosis.

\section{DISCUSSION}

Adrenal masses pose a challenge to the physician, especially when they are unintentionally discovered. An adrenal "incidentaloma" is an adrenal mass, generally 1 $\mathrm{cm}$ or more in diameter, which is discovered serendipitously during a radiologic examination performed for indications other than an evaluation for adrenal disease. ${ }^{7}$ Part of the work-up of a patient presenting with an adrenal mass is to determine whether the mass is functioning or non-functioning. A complete history and physical examination has to be done to rule out conditions such as Cushing's Syndrome, Primary Aldosteronism or Pheochromocytoma. The patient had no other known medical illnesses nor did she exhibit any sweating, palpitations, headache, weight gain, or proximal muscle weakness. Work-up for these conditions pointed to an adrenal mass that was not hyperfunctioning. The 24hour urine cortisol, plasma renin activity and plasma aldosterone concentration were within normal. The majority of cases of Primary Adrenal Lymphoma have concomitant adrenal insufficiency. Adrenal failure occurs when the parenchymal loss is more than $90 \%$ and can improve with the destruction of lymphomatous tissue at the end of a chemotherapy cycle. ${ }^{8}$ An ACTH stimulation test was performed with this patient showing inadequate adrenal reserve.

Once functionality is established, imaging studies are then done. Non-functioning adrenal tumors may either be benign or malignant. Characteristics on imaging may help to differentiate the two types. Benign masses appear as unenhanced CT attenuation of $\leq 10$ Hounsfield units or CT contrast-medium washout of $\geq 50 \%$ at 10 minutes while those that are suspicious for malignancy show $>10$ Hounsfield units on unenhanced CT attenuation or CT contrast-medium washout $<50 \%$ at 10 minutes. $^{7}$ This patient's CT scan exhibited a slight and heterogeneous enhancement following contrast infusion.

The size of the mass is also an clue for its possible nature. Current guidelines recommend adrenalectomy for masses greater than $4 \mathrm{~cm}-\mathrm{a}$ criterion which this patient fulfilled-because of increased risk for adrenal carcinoma. Adrenal carcinomas have a graver prognosis with a median survival rate of 18 months as compared to that of primary adrenal lymphoma which is as long as 34 months. $^{2}$ Because cytology cannot discriminate adrenal 
cancer from adenoma, fine-needle aspiration biopsy is usually recommended when a non-adrenal malignancy is suspected in the setting of a known tumor elsewhere, or when infection is a possibility. ${ }^{9}$ Image-guided fine-needle aspiration biopsy is relatively safe; the complication rate was $2.8 \%$ in one series of 277 biopsies. ${ }^{10}$ The risks of this procedure include adrenal hematoma, abdominal pain, hematuria, pancreatitis, pneumothorax, formation of an adrenal abscess, and tumor recurrence along the needle track. $^{7}$

After ruling out pheochromocytoma with negative hormonal testing, FNA was pursued in this case with no adverse events. Studies report a sensitivity of $81-96 \%$ and a specificity of $99-100 \%$ to identify malignant masses through fine needle aspiration biopsy. ${ }^{11}$

The patient's histopathologic findings were specific to lymphoma with characteristics of malignant, small round cells (Figures 1, 2, and 3) that stained positive for Leukocyte Common Antigen, LCA/CD45 (Figure 4) and negative for stains of synaptophysin (Figure 5), chromogranin (Figure 6) and neuron-specific enolase (Figure 7), which may be positive in neuroendocrine tumors, and was likewise negative for cytokeratin (Figure 8) which may be positive in poorly differentiated adenocarcinoma. The mass was identified to be of B cell origin due to its positivity for CD20 staining (Figure 9) and negative for CD3 (Figures 10).

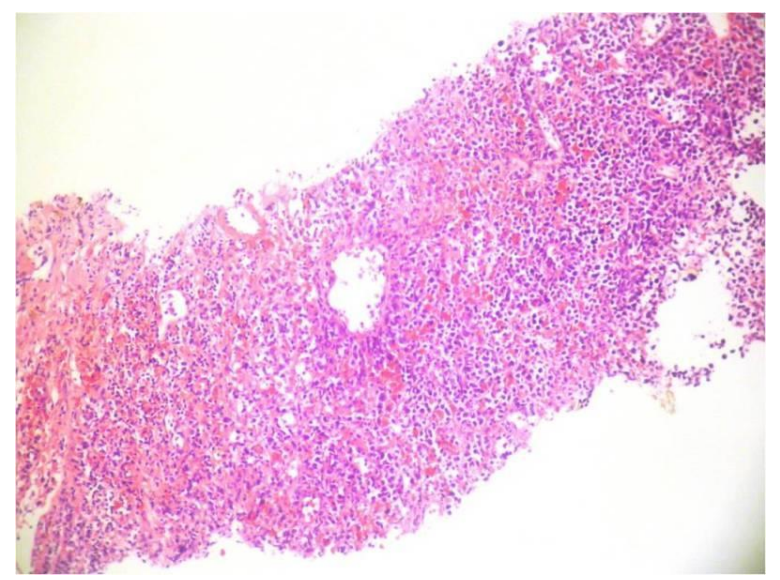

Figure 1. Tissue core showing diffuse infiltration of round blue cells.H\&E, scanning (x 4) objective.

\begin{tabular}{|c|c|c|}
\hline Lymphoma & LCA & Positive \\
\hline \multirow{5}{*}{ Neuroendocrine } & CD 20 & Positive \\
\hline & CD 3 & Negative \\
\hline & Chromogranin & Negative \\
\hline & Neuron Specific Enolase & Negative \\
\hline & Synaptophysin & Negative \\
\hline $\begin{array}{l}\text { Poorly Differentiated } \\
\text { Adenocacinoma }\end{array}$ & Cytokeratin & Negative \\
\hline
\end{tabular}

Specimen obtained via fine needle biopsy of the adrenal glands stained positive for LCA (Figure 4) and CD20 (Figure 9) and negative for CD3 (figure 10) - stains used to differentiate lymphomas from other type of malignancies; negative for synaptophysin (figure 5), chromogranin (figure 6), and NSE (figure 7) - stains for neuroendocrine tumors; negative for cytokeratin (figure 8) - stain for adenocarcinomas.

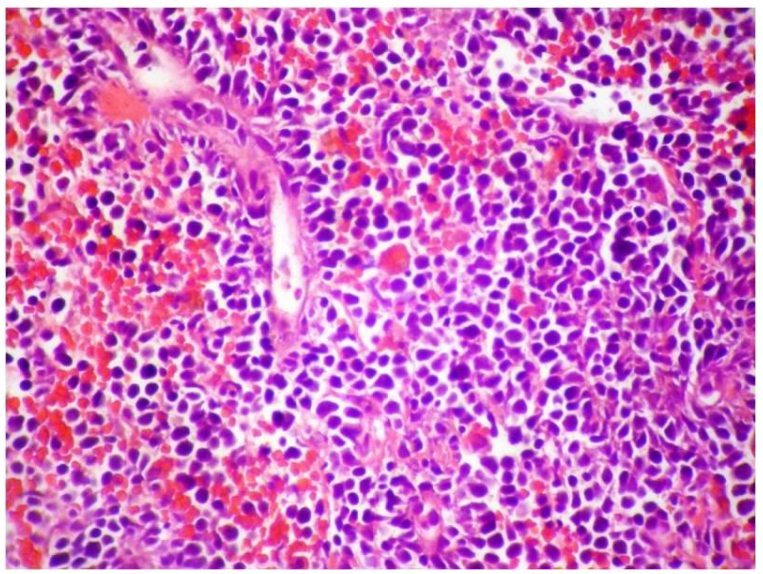

Figure 2. Diffuse infiltrate by pleomorphic nucleolated large lymphoma cells, with round nuclei and scanty cytoplasm. H\&E, x40 objective.

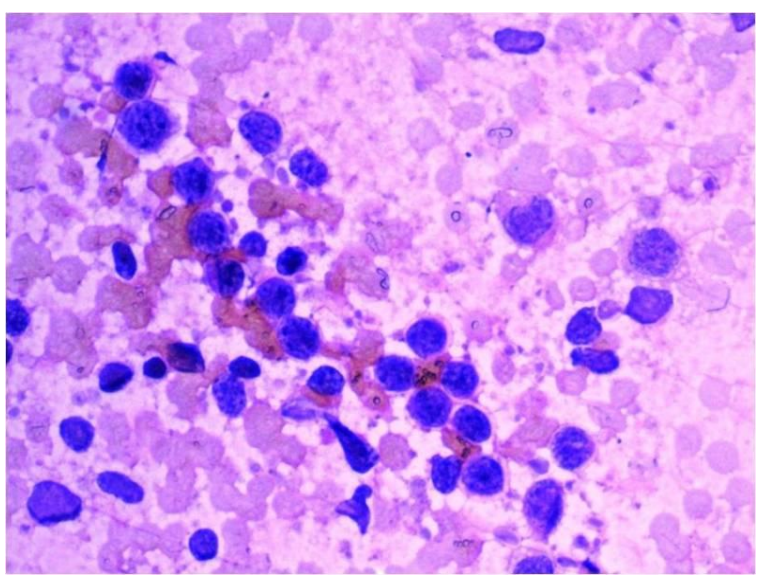

Figure 3. Imprint cytology of adrenal gland showing large lymphoma cells with prominent nucleoli and coarse chromatin. Diff-Quik Stain. X100 objective.

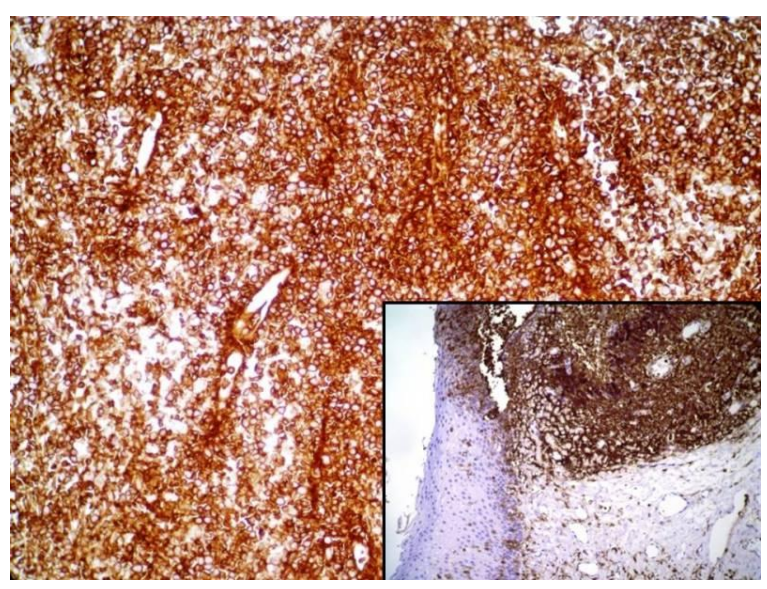

Figure 4. Adrenal gland mass showing that the lymphoma cells express LCA (Inset: Positive \& negative control, with the squamous epithelia serving as the negative control). Immunoperoxidase, x 60 objective. 


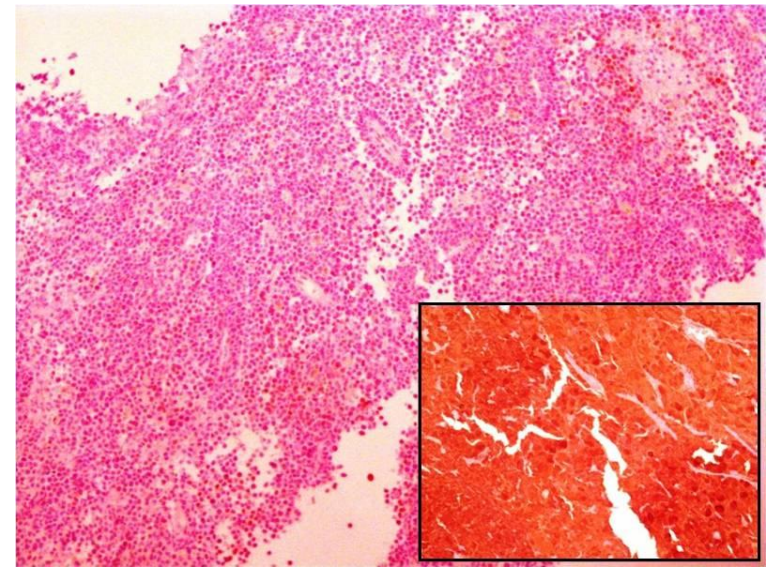

Figure 5. Adrenal gland mass showing that the lymphoma cells do not express Synaptophysin (Inset: Positive control). Immunoperoxidase, x 60 objective.

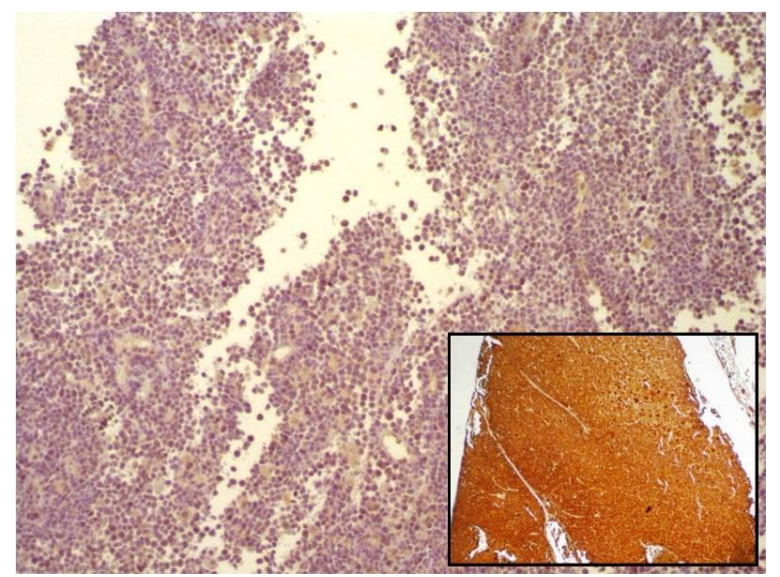

Figure 6. Adrenal mass showing that the lymphoma cells do not express Chromogranin (Inset: Positive control). Immunoperoxidase, x 60 objective.

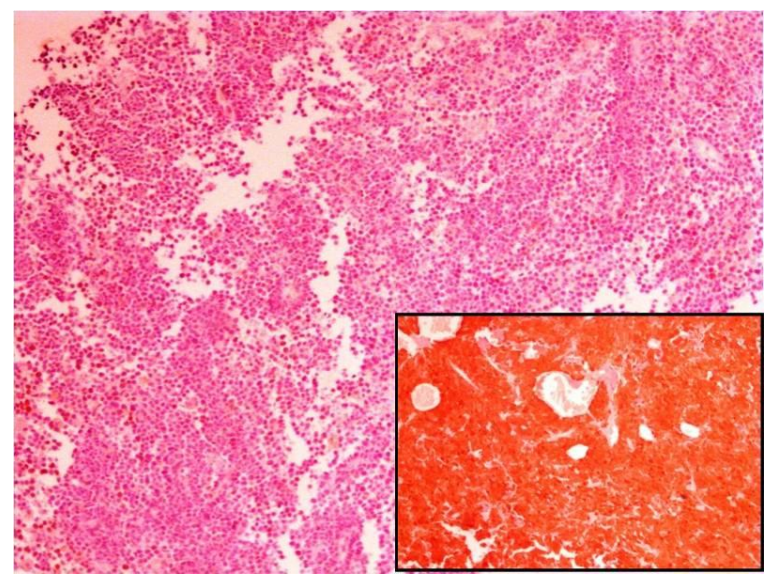

Figure 7. Adrenal gland mass showing that the lymphoma cells do not express Neuron Specific Enolase (Inset: Positive control). Immunoperoxidase, x 60 objective.

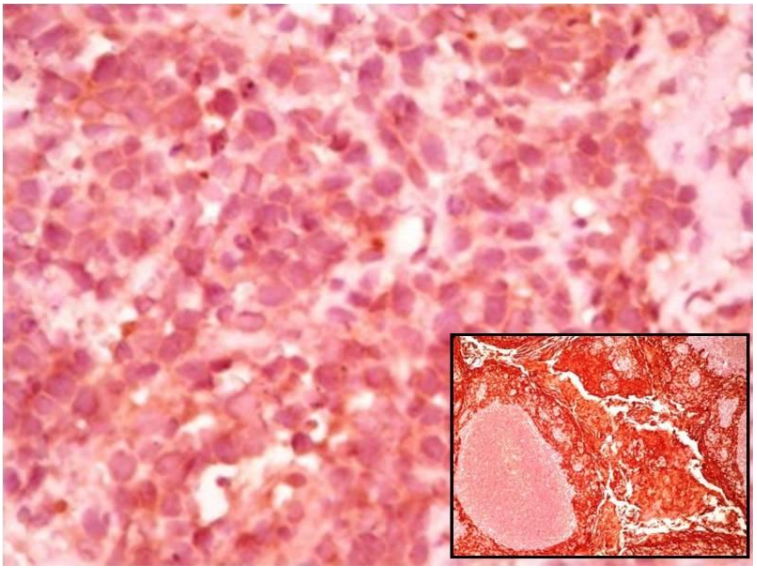

Figure 8. Adrenal gland mass showing that the lymphoma cells do not express Cytokeratin. (Inset: Positive and negative controls). Immunoperoxidase, x 60 objective.

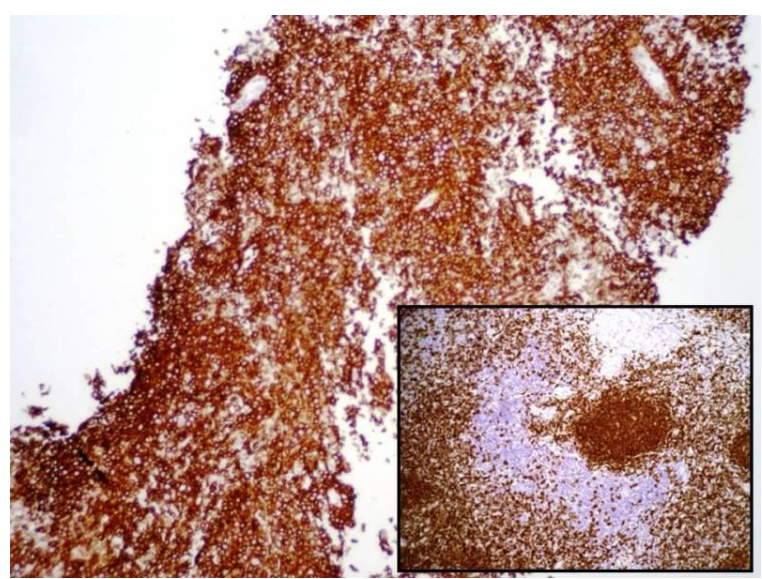

Figure 9. Adrenal gland mass showing that the lymphoma cells express CD 20 (Inset: Positive and negative controls). Immunoperoxidase, x 60 objective.

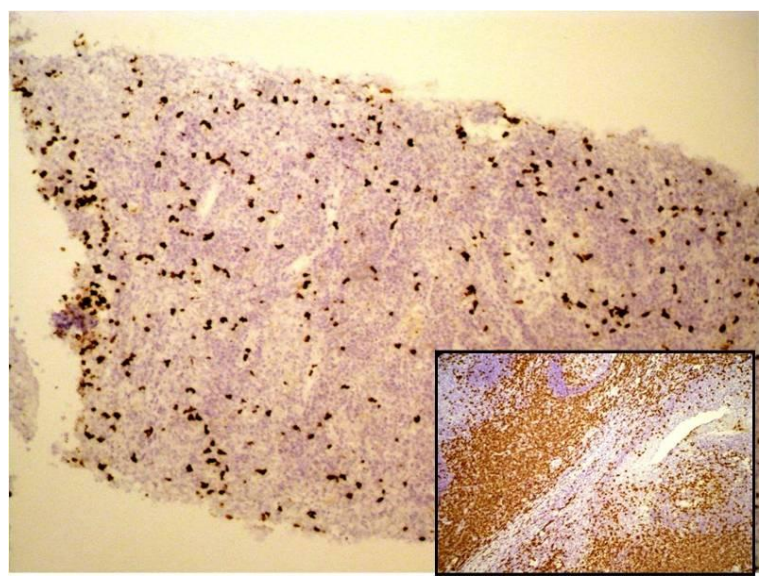

Figure 10. Adrenal gland mass showing that the lymphoma cells do not express CD3. (Inset: Positive and negative controls). Immunoperoxidase, x 60 objective. 
Why lymphoma would occur primarily in the adrenal glands without involvement of any other lymphoid tissue in the body is a mystery, as the normal human adrenals are devoid of any lymphoid or hematopoietic tissue. Proposed mechanisms for the pathophysiology of the disease include pre-existing autoimmune adrenalitis with lymphocytic infiltration or occurrence of hematopoietic rest tissue in the adrenal gland. ${ }^{12,13}$ Immune dysfunction is described to be the most important predisposing factor. ${ }^{4}$ The symptoms of the disease are variable and depend on the tumor size, the type of lymphoma or presence of adrenal insufficiency. ${ }^{3}$

Treatment of Primary Adrenal Lymphoma patients include adequate hydration and adrenocortical hormone replacement, combination chemotherapy, surgery followed by chemotherapy and/or radiation therapy and CNS prophylaxis. In general, surgery of adrenal tumors should be considered in patients with functioning cortical tumors. Regarding non-functioning masses, recommendations for treatment mainly refer to the tumor size. ${ }^{4}$ Surgical debulking of PAL in addition to chemotherapy may result in improved survival. The various regimens for chemotherapy comprise of $\mathrm{CHOP}$ (cyclophosphamide, doxorubicin, vincristine, prednisone), CHOP in combination with Rituximab, $\mathrm{CHO}$ (cyclophosphamide, doxorubicin, vincristine), CVP (vincristine, prednisone), or MACOP (cyclophosphamide, doxorubicin, prednisone, methotrexate, bleomycin). ${ }^{2}$ In one review, one third of patients achieved a partial or complete remission during chemotherapy with a mean duration of survival of $34 \pm 32$ months. ${ }^{14}$

A regimen using cyclophosphamide, vincristine and prednisone was given to this patient who opted to defer surgery due to the risk of graver morbidity. The patient survived thirteen months after diagnosis, longer than most patients with this same condition. ${ }^{5}$ With chemotherapy alone, the adrenal tumor was noted to respond with subsequent decrease in size. For patients with concomitant adrenal insufficiency, lifelong steroid replacement is warranted and at the same time may provide cure/resolution of the adrenal masses. Unfortunately for this patient, a post-therapy adrenal testing was not done to determine if the patient stayed adrenally insufficient with the resolution of the tumors.

The risk of CNS relapse has been reported to be high in primary adrenal $\mathrm{NHL}^{15}$ The incidence of CNS involvement is determined by the extent and proliferation of the disease. $^{2}$ Intracranial lesions on MRI were noted in this patient with a consideration of metastatic spread. CNS prophylaxis might be considered in primary adrenal lymphoma like other extranodal NHL. ${ }^{16} \mathrm{~A}$ poor prognosis is thought to be due to advanced age at diagnosis, large tumor size, increased LDH levels, and adrenal insufficiency at the time of presentation, indicating extensive adrenal destruction. ${ }^{15}$

\section{CONCLUSION}

A thorough investigation is warranted in cases of large adrenal masses due to a high probability of malignancy. Making an accurate diagnosis is prudent since this alters management and prognosis. Adrenocortical Carcinoma is an important differential diagnosis having a graver outcome and adrenalectomy is potentially curative in its early stages. Although the tumor's size alone was an indication for surgery, a CT scan guided FNA biopsy was pursued to first obtain a histopathologic diagnosis in this case.

Considering the patient's age, proceeding with adrenal surgery may not improve mortality but would rather increase morbidity, making chemotherapy with radiotherapy the treatment modality of choice for patients with Primary Bilateral Adrenal Lymphoma. This patient was spared surgery and its complications and underwent chemotherapy using the NHL regimen with documented decrease in the size of the adrenal masses on post therapy scans. Hormonal re-evaluation post-chemotherapy is ideal, especially with resolution of the tumors.

\section{Acknowledgements}

Dr. Janet B. Bautista from the Section of Oncology, Department of Medicine, The Medical City. Dr. Greg B. Cortez and Dr. Jose Carnate, Jr. from the Department of Surgical Pathology of The Medical City for sharing the microscopic images. Department of Radiology of The Medical City for providing the CT scan images.

\section{References}

1. Yap, $\mathrm{M}$ and Tiangco, B. Clinicopathologic profile of Filipino patients with malignant lymphomas. Phil J Internal Medicine. Nov - Dec 2004: 42: 289-296.

2. Ozimek, Alexandra, et al. Bilateral Primary Adrenal Non-Hodgkin's Lymphoma and Primary Adrenocortical Carcinoma - Review of the literature preoperative differentiation of adrenal tumors. Endocrine Journal 2008; 55 (4), 625-638.

3. Aziz, SA et al. Primary Adrenal Lymphoma: Differential involvement with varying adrenal function. Indian Journal of Endocrinology and Metabolism. $2011 ; 15$ (3).

4. Ozimek, A et al. Bilateral Primary Adrenal Non-Hodgkin's Lymphoma - A case report and review of the literature. Eur J Med Res 2008; 13: 221-228.

5. Kim et al, A case of Primary Adrenal Diffuse Large B-cell Lymphoma achieving complete remission with Rituximab-CHOP Chemotherapy. J Korean Med Sci 2009; 24: 525-8.

6. Horiguchi, $\mathrm{K}$ et al. Primary Bilateral Adrenal Diffuse Large B-cell Lymphoma demonstrating adrenal failure. Inter Med. 2010: 49; 2241-2246.

7. Young, W. The incidentally discovered adrenal mass. N Engl J Med. 2007; 356:601-10.

8. Dobrinja, C et al. Primary Bilateral Adrenal Non-Hodgkin's Burkitt-Like Lymphoma: A rare cause of primary adrenal insufficiency. Case report and literature review. Tumori. 2007; 93(6): 625-30.

9. Nieman, L. Approach to the patient with an adrenal incidentaloma. J Clin Endocrinol Metab. 2010: 95; 4106-4113.

10. Welch TJ, et al. Percutaneous adrenal biopsy: Review of a 10-year experience. Radiology. 1994; 193:341-4.

11. Terzolo, $\mathrm{M}$ et al. AME Position Statement on adrenal incidentaloma. European Journal of Endocrinology. 2011: 164; 851-870.

12. Reddy, $\mathrm{S}$ et al. Origin of primary adrenal lymphoma and predisposing factors for primary adrenal insufficiency in primary adrenal lymphoma. Indian J Endocrinol Metab. 2011 Oct-Dec; 15(4) 350-351.

13. Ellis, RD and Read, D. Bilateral adrenal non-Hodgkin's lymphoma with adrenal insufficiency. Postgrad Med J. 2000; 76:508-509.

14. Kumar, R et al. FDG-PET imaging in Primary Bilateral Adrenal Lymphoma: A case report and review of literature. Clinical Nuclear Medicine 2005; 30: 222-230.

15. Grigg, A and Connors, J. Primary Adrenal Lymphoma. Clin Lymphoma 2003; 4 154-160.

16. Kim, Y et al. Prognostic factors in primary diffuse large B-cell lymphoma of adrenal gland treated with rituximab-CHOP chemotherapy from the Consortium for Improving Survival of Lymphoma (CISL). Journal of Hematology \& Oncology 2012; 5 (49): 1-9. 